\title{
Understanding the Impact of Public Health Advice on Paediatric Healthcare Use During COVID-19: A Cross-sectional Survey of Parents
}

Emma Nicholson ( $\square$ emma.nicholson@ucd.ie)

University College Dublin https://orcid.org/0000-0002-6652-2552

Thérése McDonnell

University College Dublin

Ciara Conlon

University College Dublin

Michael Barrett

Our Lady's Children's Hospital

Fergal Cummins

University Hospital Limerick

\section{Conor Hensey}

Temple Street Children's University Hospital

Eilish McAuliffe

University College Dublin

Research article

Keywords: COVID-19, cross-sectional survey, parents, paediatric healthcare.

Posted Date: August 18th, 2020

DOI: https://doi.org/10.21203/rs.3.rs-56016/v1

License: (c) (i) This work is licensed under a Creative Commons Attribution 4.0 International License. Read Full License 


\section{Abstract}

\section{Background}

Hospital avoidance during the COVID-19 pandemic has been reported with a significant decrease in attendance at emergency departments among paediatric populations with potential increased morbidity and mortality outcomes. The present study sought to understand parents' experiences of healthcare during the initial public health stages of the COVID-19 pandemic.

\section{Methods}

A cross-sectional survey of parents of children under the age of $16(\mathrm{~N}=1044)$. The survey collected demographic information to profile parents and children, capture relevant health information such as pre-existing conditions and/or chronic illness or disability, and health service usage during the pandemic. Descriptive statistics, chi-square tests and regression analyses were used to determine the factors that influenced avoidance and hesitancy.

\section{Results}

$23 \%$ of parents stated that they were much more hesitant to access health services upon implementation of the initial public health restrictions. Parents with a higher perception of risk of their children contracting COVID-19 $(\chi 2(3)=33.8618, p<0.000)$, and stronger levels of concern regarding the effects of COVID-19 $(\chi 2(3)=23.9189, p<0.000)$ were more likely to be hesitant. Stress also appeared to be a factor in hesitancy with higher than normal stress levels significantly associated with hesitancy (RRR= 2.31, Cl: $1.54-3.47)$, while those with severe/extremely severe stress were over three times more likely to be hesitant (RRR:3.37, Cl:1.81 - 6.27). Approximately one third of the sample required healthcare for their children during the public health restrictions to delay the spread of COVID-19, however, one in five of these parents avoided accessing such healthcare when needed. Of those that required healthcare, parents who avoided were more likely to report that the services were needed more by others $(\chi 2$ (1) 20.3470, $p<0.000)$. Those who felt that the government advice was to stay away from health services were 1.7 times more likely to be much more hesitant (RRR:1.71, Cl; $1.10-2.67$ ).

\section{Conclusion}

The misinterpretation of government public health advice, stress and the perception of risk each contributed to parental avoidance of or hesitancy to utilize healthcare services during the public health measures imposed to combat COVID-19.

\section{Background}

The most effective public health response to delay and reduce the spread of COVID-19 appears to be widespread restrictions on movement and physical interaction (1). These restrictions included the closure of schools, sporting, social and cultural activities and institutions, banning of large gatherings of people and the closure of non-essential workplaces. In many countries such public health measures have never been implemented at a national level and therefore, the impact of the measures on public behaviour is unknown. The public health response to delay the spread of SARS in Canada resulted in widespread community challenges such as unwarranted avoidance of public spaces, and it has been suggested that more appropriate public health advice may have reduced this avoidance behaviour (2). Moreover, during the H1N1 outbreak in Hong Kong, approximately two thirds of people avoided going to hospital (Lau et al., 2010), and a hypothetical study examining public perception of risk during an influenza pandemic, found that approximately $40 \%$ of respondents stated that they would avoid hospital for fear of infection (3). Negative psychological outcomes have been associated with being placed in quarantine during previous disease outbreaks such as SARS, with inadequate information from public health authorities found to have exacerbated stress levels (Brooks et al, 2020).

Understanding the behaviour of populations during a pandemic is key to ensuring the maintenance of health during future public health emergencies and in the instance of a second wave of COVID-19. Hospital avoidance during the COVID-19 pandemic has been reported with a significant decrease in attendance at emergency departments among paediatric populations with potential increased morbidity and mortality outcomes $(4,5)$. While this decrease can be partially explained by fewer social interactions resulting in a lower risk of contracting some infectious diseases as well as a reduction in minor accidents and injuries, it is also likely as a result of a shift in health-seeking behaviour of parents $(6,7)$. Of particular concern are children with pre-existing conditions who may be at increased risk due to the impact of delays in seeking healthcare $(4,8)$.

The aim of the study was to assess avoidance behaviour and the level of hesitancy by parents on accessing healthcare for their child during the COVID-19 pandemic and to determine the prevalence and factors associated with healthcare avoidance.

\section{Method}

\section{Survey Design}

A cross-sectional survey was designed to capture the experience of parents accessing healthcare for their child during the COVID-19 pandemic. The survey collected demographic information to profile parents and children and capture relevant health information such as pre-existing conditions and/or chronic illness or disability. Experiences of COVID-19 and self-isolation were also assessed. Hesitancy in accessing healthcare services, use and/or avoidance of unscheduled healthcare services for their children at two distinct stages of the public health response. both the initial "Delay and Mitigation" phases or "lockdown" as it was known within public discourse, when schools, businesses and travel restrictions were put in place (beginning on the $12^{\text {th }}$ of March) and "Phase One" of reopening, the first stage of official easing of restrictions ( $18^{\text {th }}$ of May). Figure 1 provides a detailed outlined of the public health stages of the 
COVID-19 response. The stress scale from the Depression Anxiety Stress Scale (DASS-21) (9) was also included in the survey. The survey was delivered online through Qualtrics ${ }^{\mathrm{TM}}$ and is available as Additional File 1.

\section{Participants}

Parents of children under the age of 16 living in Ireland $(\mathrm{N}=1044)$ were recruited to the study, of which $62 \%$ were female, $83.7 \%$ were either married or cohabiting and $52.7 \%$ had a degree or postgraduate qualification. $53.1 \%$ had a child or children aged four or under, $52 \%$ had children aged 10 to 16 , and $60.4 \%$ had more than one child. A comprehensive geographical spread was achieved, with each county of Ireland represented. $46 \%$ of participants reported that a person in their household had an underlying health condition, $34 \%$ of whom were children under $16.1 .3 \%$ of participants $(n=11)$ indicated they or another household member were confirmed cases of COVID-19, while $25 \%$ of participants stated they or another household member self-isolated due to COVID-19. The number of respondents who held a medical card which provides access to medical services, prescription medicines and hospital care free of charge (38\%), which grants the holder access to healthcare services free of charge to those on low income or with a specified illness, is slightly higher than the national proportion in 2018 (32.4\%) (10), however, the proportion of private health insurance holders accurately reflects the current national average (46.2\%) (11). Demographic information pertaining to participants can be found in Table 1. 
Table 1

Characteristics of Sample

\begin{tabular}{|c|c|c|c|c|}
\hline & & $N=1,044$ & Total \% (n) & \\
\hline Gender of parent & Female & & $62.1(648)$ & \\
\hline \multirow[t]{4}{*}{ Age of parent } & $<29$ & & $12.0(125)$ & \\
\hline & $30-39$ & & $41.2(430)$ & \\
\hline & $40-49$ & & $37.2(388)$ & \\
\hline & $50>$ & & $9.7(101)$ & \\
\hline \multirow[t]{4}{*}{ Marital Status } & Single & & $10.6(111)$ & \\
\hline & Married & & $68.2(712)$ & \\
\hline & Cohabiting & & $15.5(162)$ & \\
\hline & Divorced/Separated/Widowed & & $5.7(59)$ & \\
\hline \multirow[t]{4}{*}{ Location } & Dublin & & $27.2(284)$ & \\
\hline & Cork & & $10.8(113)$ & \\
\hline & Galway & & $6.9(72)$ & \\
\hline & Rest of country & & $55.1(575)$ & \\
\hline \multirow[t]{4}{*}{ Age of Children } & $<2$ & & $23.1(241)$ & \\
\hline & $2-4$ & & $30.0(313)$ & \\
\hline & $5-9$ & & $45.9(479)$ & \\
\hline & $10-16$ & & $52.0(543)$ & \\
\hline \multirow[t]{3}{*}{ No. of children } & One child & & $39.6(413)$ & \\
\hline & Two children & & $38.9(406)$ & \\
\hline & Three or more children & & $21.6(225)$ & \\
\hline \multirow[t]{4}{*}{ Education } & Lower Secondary & & $4.5(47)$ & \\
\hline & Upper Secondary & & $14.0(146)$ & \\
\hline & Post-Secondary certificate /vocational & & $28.8(301)$ & \\
\hline & Degree or Postgraduate & & $52.7(550)$ & \\
\hline \multirow[t]{5}{*}{ Health cover status } & No cover & & $18.5(193)$ & \\
\hline & Medical card only & & $27.8(290)$ & \\
\hline & GP visit card only & & $8.1(84)$ & \\
\hline & Private Insurance only & & $35.5(371)$ & \\
\hline & Both insurance \& medical card & & $10.2(106)$ & \\
\hline Profession & Healthcare professional & & $10.6(111)$ & \\
\hline \multirow[t]{3}{*}{ Underlying Health Condition } & Underlying health conditions in household & & $46.4(484)$ & \\
\hline & Child under 16 with underlying health condition & & $15.8(165)$ & \\
\hline & Other household member with underlying health condition & & $37.9(396)$ & \\
\hline \multirow[t]{8}{*}{ Type of conditions: } & & & $\begin{array}{l}\text { Child under } \\
16\end{array}$ & $\begin{array}{l}\text { Other household } \\
\text { member }\end{array}$ \\
\hline & Cancer & & $0.6(6)$ & $3.5(37)$ \\
\hline & Chronic lung condition e.g. Asthma & & $9.0(94)$ & $11.8(123)$ \\
\hline & Diabetes & & $1.2(12)$ & $7.9(82)$ \\
\hline & High blood pressure/hypertension & & $0.4(4)$ & $18.4(192)$ \\
\hline & Immunocompromising condition & & $2.3(24)$ & $12.2(127)$ \\
\hline & Intellectual disability & & $4.9(51)$ & $2.2(23)$ \\
\hline & Heart/kidney/liver disease or organ transplant & & $1.2(12)$ & $2.9(30)$ \\
\hline
\end{tabular}




\begin{tabular}{|c|c|c|}
\hline \multirow{7}{*}{$\begin{array}{l}\text { Concerns about accessing healthcare } \\
\text { during COVID }\end{array}$} & Worried about catching COVID- 19 & $67.6(706)$ \\
\hline & $\begin{array}{l}\text { Worried about being judged for using a service if it wasn't an } \\
\text { emergency }\end{array}$ & $24.4(255)$ \\
\hline & Thought the service would be needed more urgently by other people & $25.2(263)$ \\
\hline & $\begin{array}{l}\text { Thought the government advice meant I couldn't go to the GP or } \\
\text { hospital }\end{array}$ & $17.5(183)$ \\
\hline & $\begin{array}{l}\text { Worried that the service would be extremely busy and would have to } \\
\text { wait for too long }\end{array}$ & $30.2(315)$ \\
\hline & Don't have a car and didn't want to use public transport & $9.1(95)$ \\
\hline & Something else & $8.8(92)$ \\
\hline \multirow[t]{5}{*}{ DASS Stress } & Normal & $65.0(679)$ \\
\hline & Mild & $12.7(133)$ \\
\hline & Moderate & $13.2(138)$ \\
\hline & Severe & 7.2(75) \\
\hline & Extremely severe & $1.8(19)$ \\
\hline
\end{tabular}

\section{Sampling and data collection}

Participants were recruited using Qualtrics ${ }^{\mathrm{TM}}$ market research panels which collects data from a diverse network of respondents. Respondents were randomly selected for the survey based on their existing profile which for this study was parents with children under the age of 16 in Ireland. Participants received a small incentive for taking part. Data was collected from 5/6/2020 to 10/6/2020 during Phase One of easing restrictions.

\section{Data Analysis}

Descriptive analyses were used to assess the demographic characteristics of the sample, personal experience of COVID-19, concerns held by participants, sources of information, the prevalence of healthcare avoidance by parents during the pandemic, and levels of hesitancy at two timepoints (the Delay \& Mitigation phases and Phase One of reopening). Chi-square ( $\chi 2$ ) tests were used to examine the statistical significance between key demographics, reported hesitancy and avoidance of health services. Logistic regression was used to establish the factors that predicted reported hesitancy and avoidance behaviour. The sample size $(\mathrm{N}=1044)$ was selected to provide a representative proportion of the target population. The analyses were carried out in STATA 15.0 .

\section{Results}

\section{Experience of COVID-19 and perception of risk}

$25 \%(n=261)$ of the sample indicated they had self-isolated due to the COVID-19 pandemic, while 1.3\% $(n=14)$ of participants or another household member had contracted COVID-19. Almost half of participants were slightly concerned (46\%) of the risk of their child contracting COVID-19, 32\% were moderately concerned while $9 \%$ felt their child was at high risk. $50 \%$ said their child would be somewhat affected if they contracted COVID-19, while $28 \%$ said they would be quite unwell and $10 \%$ thought their child would be very unwell.

\section{Sources of information and concerns about accessing healthcare}

Participants were asked where they sought information on accessing healthcare for their child following the onset of the pandemic. The majority (88.5\%) utilized either official government or Health Service Executive (HSE) sources. 18\% accessed information through friends and family on social media, while $17 \%$ turned to 'experts' on social media.

Participants were asked to select the reasons that caused the greatest amount of concern when considering accessing healthcare for their child during COVID19. Fear of contracting COVID-19 (67\%) was the most commonly reported concern, while $30 \%$ stated that they were concerned the service would be busy at this time. $25 \%$ believed that services were needed more by others while $24 \%$ feared they would be judged for attending. $18 \%$ reported that they thought the public health advice issued by the government was to avoid health services.

Regarding the $18 \%$ who perceived that official advice administered by authorities meant health services were to be avoided ( $n=183$ ), a higher proportion of this subgroup also reported significantly higher levels of stress $(\chi 2(2)=13.3163, p<0.01)$ and were much more or somewhat more hesitant about accessing healthcare for their children in the Delay and Mitigation phase $(\chi 2(3)=11.4103, p<0.05)$. Further details on this group can be found in Additional File 2 .

\section{Hesitancy regarding access of healthcare}


Participants were asked their level of hesitancy about accessing healthcare, both during the Delay and Mitigation or "lockdown" stage and Phase One of reopening stage, by selecting one of five points on a scale ranging from much more to much less. $23 \%$ stated that they were much more hesitant and $35 \%$ were somewhat more hesitant during Delay and Mitigation, however this reduced to $5 \%$ and $18 \%$ respectively during Phase One. $34 \%$ felt about the same regarding accessing healthcare during Delay and Mitigation, increasing to $47 \%$ during Phase one. $19 \%$ of participants indicated they were hesitant at both stages.

Chi-square $(\chi 2)$ tests were used to establish between group differences in reported hesitancy and are reported in Table 2. Female participants were significantly more likely to report being hesitant to attend health services during the Delay and Mitigation period $(\chi 2(3)=19.7608, p<0.000)$ and this was also significant at Phase One. Parents of children who had an underlying health condition were also more likely to report being much more hesitant and somewhat more hesitant compared to those without $(\chi 2(3)=10.6619, p=0.014)$. This effect was not significant at Phase One. Significant associations were also found between parents who reported higher stress levels (i.e., scored severe/extremely severe on the DASS) $(\chi 2(6)=47.2862, p<.000$.), parents with a higher perception of risk of their children contracting COVID-19 $(\chi 2(3)=33.8618, p<0.000)$, and stronger levels of concern regarding the effect COVID-19 would have on their children $(\chi 2(3)=23.9189, p<0.000)$. Finally, greater hesitancy was reported by those who thought that government advice was to stay away from health services $(\chi 2(3)=11.4103, p=0.01)$. Each of these effects were also significant in Phase One, as can be seen in Table 2. 


\begin{tabular}{|c|c|c|c|c|c|c|c|c|c|c|}
\hline & \multicolumn{5}{|c|}{ Delay \& Mitigation Period } & \multicolumn{5}{|c|}{ Phase One of reopening } \\
\hline & $\begin{array}{l}\text { Much } \\
\text { more }\end{array}$ & $\begin{array}{l}\text { Somewhat } \\
\text { more }\end{array}$ & $\begin{array}{l}\text { About the } \\
\text { same }\end{array}$ & $\begin{array}{l}\text { Somewhat } \\
\text { or much } \\
\text { less }\end{array}$ & $\begin{array}{l}p- \\
\text { value }\end{array}$ & $\begin{array}{l}\text { Much } \\
\text { more }\end{array}$ & $\begin{array}{l}\text { Somewhat } \\
\text { more }\end{array}$ & $\begin{array}{l}\text { About the } \\
\text { same }\end{array}$ & $\begin{array}{l}\text { Somewhat } \\
\text { or much } \\
\text { less }\end{array}$ & $\begin{array}{c}\mathrm{p}- \\
\text { valu€ }\end{array}$ \\
\hline & $\%(n)$ & $\%(n)$ & $\%(n)$ & $\%(n)$ & & $\%(n)$ & $\%(n)$ & $\%(n)$ & $\%(n)$ & \\
\hline Total & $23.2(242)$ & $35.1(366)$ & $34.2(357)$ & $7.6(79)$ & & $5.2(54)$ & $17.6(184)$ & $46.8(489)$ & $30.4(317)$ & \\
\hline Female & $71.9(174)$ & $63.7(233)$ & $56.3(201)$ & $50.6(40)$ & $\begin{array}{l}\mathrm{p}< \\
0.000\end{array}$ & $62.7(34)$ & $62.5(115)$ & $57.7(282)$ & $68.5(217)$ & $\begin{array}{l}p= \\
0.02=\end{array}$ \\
\hline \multicolumn{11}{|l|}{ Age of parent } \\
\hline$<29$ & 13.2(32) & $10.9(40)$ & $11.5(41)$ & 15.2(12) & \multirow{4}{*}{$\begin{array}{l}p= \\
0.916\end{array}$} & $22.2(12)$ & $12.5(23)$ & $12.5(61)$ & $9.2(29)$ & \\
\hline $30-39$ & $41.3(100)$ & $42.1(154)$ & $39.2(140)$ & $45.6(36)$ & & $33.3(18)$ & 49.5(91) & $40.3(197)$ & $39.1(124)$ & $\begin{array}{l}p= \\
0.035\end{array}$ \\
\hline $40-49$ & $36.0(87)$ & $37.7(138)$ & $38.7(138)$ & $31.7(25)$ & & $35.2(19)$ & $29.9(55)$ & $38.7(189)$ & $39.4(125)$ & \\
\hline $50>$ & $9.5(23)$ & $9.3(34)$ & 10.6(38) & $7.6(6)$ & & $9.3(5)$ & $8.2(15)$ & $8.6(42)$ & 12.3(39) & \\
\hline \multicolumn{11}{|l|}{ Marital Status } \\
\hline Single & $12.8(31)$ & $8.5(31)$ & $11.5(41)$ & 10.1(8) & \multirow{4}{*}{$\begin{array}{l}p= \\
0.429\end{array}$} & 11.1(6) & $11.4(21)$ & $10.4(51)$ & 10.4(33) & \\
\hline Married & $69.4(168)$ & $70.8(259)$ & $65.0(232)$ & $67.1(53)$ & & $61.1(33)$ & $71.7(132)$ & $67.3(329)$ & $68.8(218)$ & $\begin{array}{l}p= \\
0.73 c\end{array}$ \\
\hline Cohabiting & $12.0(29)$ & 16.1(59) & 16.5(59) & 19.0(15) & & $18.5(10)$ & $13.0(24)$ & $17.0(83)$ & $14.2(45)$ & \\
\hline Divorced/Separated/Widowed & $5.8(14)$ & $4.6(17)$ & $7.0(25)$ & $3.8(3)$ & & $9.3(5)$ & $3.8(7)$ & $5.3(26)$ & $6.6(21)$ & \\
\hline \multicolumn{11}{|l|}{ No. of children } \\
\hline One child & $39.7(96)$ & $35.0(128)$ & $46.5(166)$ & $29.1(23)$ & \multirow{3}{*}{$\begin{array}{l}p= \\
0.003\end{array}$} & $42.6(23)$ & $40.8(75)$ & $42.1(206)$ & $34.4(109)$ & \\
\hline Two children & $39.3(95)$ & $39.3(144)$ & $37.8(135)$ & $40.5(32)$ & & $38.9(21)$ & $37.5(69)$ & $38.5(188)$ & $40.4(128)$ & $\begin{array}{l}p= \\
0.34 c\end{array}$ \\
\hline Three or more children & $21.1(51)$ & 25.7(94) & $15.7(56)$ & $30.4(24)$ & & $18.5(10)$ & $21.7(40)$ & 19.4(95) & $25.2(80)$ & \\
\hline \multicolumn{11}{|l|}{ Education } \\
\hline Lower Secondary & $2.9(7)$ & $2.7(10)$ & $6.2(22)$ & 10.1(8) & \multirow{4}{*}{$\begin{array}{l}p= \\
0.080\end{array}$} & $1.9(1)$ & $2.2(4)$ & $4.7(23)$ & $6.0(19)$ & \\
\hline Upper Secondary & 15.3(37) & $14.2(52)$ & $12.3(44)$ & $16.5(13)$ & & $13.0(7)$ & $15.8(29)$ & $13.5(66)$ & $13.9(44)$ & $\begin{array}{l}p= \\
0.78 €\end{array}$ \\
\hline $\begin{array}{l}\text { Post-Secondary certificate } \\
\text { /vocational }\end{array}$ & $27.7(67)$ & $31.1(111)$ & $16.5(13)$ & $26.6(21)$ & & $29.6(16)$ & $28.3(52)$ & $28.8(141)$ & 29.0(92) & \\
\hline Degree or Postgraduate & $54.1(131)$ & $50.4(180)$ & $14.0(146)$ & $46.8(37)$ & & $55.6(30)$ & $53.8(99)$ & $53.0(259)$ & $51.1(162)$ & \\
\hline \multicolumn{11}{|l|}{ Health cover status } \\
\hline No cover & $16.9(41)$ & $17.8(65)$ & $21.6(77)$ & $12.7(10)$ & \multirow{5}{*}{$\begin{array}{l}p= \\
0.083\end{array}$} & 11.1(6) & 16.8(31) & 18.8(92) & $20.2(64)$ & \\
\hline Medical card only & $26.0(63)$ & 25.1(92) & $28.6(102)$ & $41.8(33)$ & & 24.1(13) & $28.3(52)$ & $30.3(148)$ & $24.3(77)$ & $\begin{array}{l}p= \\
0.115\end{array}$ \\
\hline GP visit card only & $8.7(21)$ & $8.7(32)$ & $6.4(23)$ & 10.1(8) & & $3.7(2)$ & $9.2(17)$ & $8.8(43)$ & $6.9(22)$ & \\
\hline Private Insurance only & $38.0(92)$ & $36.9(135)$ & $35.3(126)$ & $22.8(18)$ & & $42.6(23)$ & $32.6(60)$ & $32.9(161)$ & $40.1(127)$ & \\
\hline $\begin{array}{l}\text { Both insurance \& medical } \\
\text { card }\end{array}$ & $10.3(25)$ & $11.5(42)$ & $8.1(29)$ & $12.7(10)$ & & $18.5(10)$ & $13.0(24)$ & $9.2(45)$ & $8.5(27)$ & \\
\hline Healthcare professional & $9.9(24)$ & $11.2(41)$ & 10.1(36) & $12.7(10)$ & $\begin{array}{l}p= \\
0.871\end{array}$ & 16.7(9) & $12.5(23)$ & $10.2(50)$ & $9.2(29)$ & $\begin{array}{l}p= \\
0.312\end{array}$ \\
\hline \multicolumn{11}{|c|}{ Underlying health condition in household } \\
\hline Yes & $53.3(129)$ & $46.5(170)$ & $42.0(150)$ & 44.3(35) & \multirow[t]{2}{*}{$\begin{array}{l}p= \\
0.057\end{array}$} & $38.9(21)$ & $48.9(90)$ & $45.2(221)$ & $48.0(152)$ & $\begin{array}{l}p= \\
0.51 \epsilon\end{array}$ \\
\hline No & $46.7(113)$ & $54.6(196)$ & $58.0(207)$ & $55.7(44)$ & & $61.1(33)$ & $51.1(94)$ & $54.8(268)$ & $52.1(165)$ & \\
\hline
\end{tabular}


Child under 16 with underlying health condition

\begin{tabular}{|c|c|c|c|c|c|c|c|c|c|c|}
\hline Yes & $20.7(50)$ & $17.5(64)$ & $11.5(41)$ & $12.7(10)$ & \multirow[t]{2}{*}{$\begin{array}{l}p= \\
0.014\end{array}$} & $9.3(5)$ & $16.9(31)$ & $16.4(80)$ & $15.5(49)$ & $\begin{array}{l}p= \\
0.56 \epsilon\end{array}$ \\
\hline No & $\begin{array}{l}79.3 \\
(192)\end{array}$ & $82.5(302)$ & $\begin{array}{l}88.5 \\
(316)\end{array}$ & $87.3(69)$ & & $90.7(49)$ & $83.62(153)$ & $\begin{array}{l}83.6 \\
(409)\end{array}$ & $84.5(268)$ & \\
\hline \multicolumn{11}{|c|}{ Member of household isolated } \\
\hline Yes & $29.8(72)$ & 26.8(98) & $21.0(75)$ & $20.3(16)$ & \multirow[t]{2}{*}{$\begin{array}{l}p= \\
0.057\end{array}$} & 29.6(16) & $29.9(55)$ & 23.3(114) & $24.0(76)$ & $\begin{array}{l}p= \\
0.274\end{array}$ \\
\hline No & $70.2(170)$ & $73.2(268)$ & $79.0(282)$ & $79.8(63)$ & & $70.4(38)$ & 70.1(129) & 76.7(375) & $76.0(241)$ & \\
\hline \multicolumn{11}{|l|}{ Was healthcare needed } \\
\hline Yes, accessed healthcare & $33.1(80)$ & $30.1(110)$ & 19.3(69) & $26.6(21)$ & \multirow[t]{3}{*}{$\begin{array}{l}\mathrm{p}< \\
0.000\end{array}$} & $27.8(15)$ & $32.6(60)$ & 23.5(115) & $28.4(90)$ & $\begin{array}{l}p= \\
0.00 \Xi\end{array}$ \\
\hline $\begin{array}{l}\text { Yes, but didn't access } \\
\text { healthcare }\end{array}$ & $10.3(25)$ & $8.7(32)$ & $3.9(14)$ & $11.4(9)$ & & $16.7(9)$ & $9.8(18)$ & $5.5(27)$ & $8.2(26)$ & \\
\hline No, not needed & $56.6(137)$ & $61.2(224)$ & $76.8(274)$ & $62.0(49)$ & & $55.6(30)$ & $57.6(106)$ & $71.0(347)$ & $63.4(201)$ & \\
\hline \multicolumn{11}{|c|}{ Perception of Risk of child contracting COVID-19 } \\
\hline High risk & $16.5(40)$ & $5.7(21)$ & $7.8(28)$ & $11.4(9)$ & \multirow[t]{4}{*}{$\begin{array}{l}\mathrm{p}< \\
0.000\end{array}$} & 22.2(12) & $12.5(23)$ & 7.8(38) & $7.9(25)$ & $\begin{array}{l}\mathrm{p}< \\
0.00 c\end{array}$ \\
\hline Moderately concerned & $38.4(93)$ & $32.2(118)$ & $24.7(88)$ & $38.0(30)$ & & $44.4(24)$ & $40.2(74)$ & $27.4(134)$ & $30.6(97)$ & \\
\hline Slightly concerned & $36.0(87)$ & $50.6(185)$ & $49.9(178)$ & $32.9(26)$ & & 22.2(12) & $35.9(66)$ & $50.9(249)$ & $47.0(149)$ & \\
\hline Not at all & $9.1(22)$ & $11.5(42)$ & $17.7(63)$ & $17.7(14)$ & & 11.1(6) & $11.4(21)$ & $13.9(68)$ & $14.5(46)$ & \\
\hline \multicolumn{11}{|c|}{ Perception of effect of COVI-19 on child } \\
\hline Very unwell & 15.7(38) & $5.5(20)$ & $8.7(31)$ & $17.7(14)$ & \multirow{4}{*}{$\begin{array}{l}\mathrm{p}< \\
0.000\end{array}$} & 24.1(13) & $7.6(14)$ & $9.0(44)$ & 10.1(32) & $\begin{array}{l}\mathrm{p}< \\
0.00 c\end{array}$ \\
\hline Quite unwell & $35.1(85)$ & $29.0(106)$ & $23.8(85)$ & 24.1(19) & & $27.8(15)$ & $37.5(69)$ & $28.8(141)$ & $22.1(70)$ & \\
\hline Somewhat unwell & $40.9(99)$ & 54.1(198) & $51.5(184)$ & $45.6(36)$ & & $37.0(20)$ & $42.4(78)$ & $48.7(238)$ & $57.1(181)$ & \\
\hline Unaffected & $8.3(20)$ & $11.5(42)$ & $16.0(57)$ & 12.7(10) & & 11.1(6) & $12.5(23)$ & 13.5(66) & 10.7(34) & \\
\hline \multicolumn{11}{|l|}{ DASS Stress } \\
\hline Normal & 49.2(119) & 65.3(239) & $74.5(266)$ & $69.6(55)$ & \multirow[t]{5}{*}{$\begin{array}{l}\mathrm{p}< \\
0.000\end{array}$} & $37.0(20)$ & $53.8(99)$ & $71.6(350)$ & $66.3(210)$ & $\begin{array}{l}\mathrm{p}< \\
0.00 c\end{array}$ \\
\hline Mild & 16.1(39) & $12.8(47)$ & 10.9(39) & 10.1(8) & & 16.7(9) & 18.5(34) & 10.8(53) & 11.7(37) & \\
\hline Moderate & $18.2(44)$ & $13.9(51)$ & $9.2(33)$ & $12.7(10)$ & & 22.2(12) & $15.8(29)$ & $10.4(51)$ & $14.5(46)$ & \\
\hline Severe & $12.8(31)$ & $6.3(23)$ & $4.2(15)$ & $7.6(6)$ & & 22.2(12) & $8.7(16)$ & $5.5(27)$ & $6.3(20)$ & \\
\hline Extremely severe & $3.7(9)$ & $1.6(6)$ & $1.1(4)$ & $0.0(0)$ & & $1.9(1)$ & $3.3(6)$ & $1.6(8)$ & $1.3(4)$ & \\
\hline
\end{tabular}

\section{Healthcare during COVID-19}

$34 \%$ of participants stated that their child required healthcare during the pandemic, of whom $22 \%(n=80)$ decided against seeking healthcare. Of those that did, $42 \%$ had a face-to face consultation with a general practitioner (GP) or through private facilities, while $39 \%$ availed of a remote consultation by phone or video. $13 \%$ of those that accessed healthcare did so at an emergency department (ED). Table 3 displays the characteristics of those who needed healthcare, split by whether or not healthcare was accessed. Chi-square $(\chi 2)$ tests were utilized to identify differences between those who accessed healthcare and those who decided against. $66.1 \%$ of those who attended services were concerned about their child contracting COVID- 19 while $54 \%$ of those that avoided healthcare reported this concern $(\chi 2$ (1) $4.0678, p=0.04$ ), while $43 \%$ were concerned that the service was needed more by others compared to $18.2 \%$ of those who attended $(\chi 2$ (1) 20.3470, $p<0.000)$. No other significant difference between the groups was found. 


\begin{tabular}{|c|c|c|c|c|c|c|}
\hline \multicolumn{7}{|l|}{$N=360$} \\
\hline & & \multirow{2}{*}{\multicolumn{2}{|c|}{$\begin{array}{l}\text { Accessed healthcare } \\
\%(n)\end{array}$}} & \multirow{2}{*}{\multicolumn{2}{|c|}{$\begin{array}{l}\text { Needed healthcare } \\
\text { but avoided } \\
\%(n)\end{array}$}} & \multirow[t]{2}{*}{ p-value } \\
\hline & & & & & & \\
\hline \multicolumn{2}{|l|}{ Needed healthcare for child } & \multicolumn{2}{|l|}{$77.8(280)$} & \multicolumn{2}{|l|}{$22.2(80)$} & \\
\hline \multicolumn{7}{|l|}{ Type of healthcare accessed } \\
\hline & Face to face (GP/Private service) & \multicolumn{2}{|l|}{$41.9(111)$} & \multicolumn{2}{|l|}{$\mathrm{n} / \mathrm{a}$} & \\
\hline & Video/Phone & \multicolumn{2}{|l|}{$39.3(104)$} & \multicolumn{2}{|l|}{$\mathrm{n} / \mathrm{a}$} & \\
\hline & $E D$ & \multicolumn{2}{|l|}{$13.6(36)$} & \multicolumn{2}{|l|}{$\mathrm{n} / \mathrm{a}$} & \\
\hline & Other & \multicolumn{2}{|l|}{$5.3(14)$} & \multicolumn{2}{|l|}{$\mathrm{n} / \mathrm{a}$} & \\
\hline Gender of parent & Female & $66.8(187)$ & & $58.8(47)$ & & $\begin{array}{l}p= \\
0.184\end{array}$ \\
\hline \multirow[t]{4}{*}{ Age of parent } & $<29$ & $16.8(47)$ & & $18.8(15)$ & & \multirow[t]{4}{*}{$p=0.587$} \\
\hline & $30-39$ & $48.9(137)$ & & $46.3(37)$ & & \\
\hline & $40-49$ & $25.4(71)$ & & $30.0(24)$ & & \\
\hline & $50>$ & $8.9(25)$ & & $5.0(4)$ & & \\
\hline \multirow[t]{4}{*}{ Marital Status } & Single & $8.2(23)$ & & $8.8(7)$ & & \multirow[t]{4}{*}{$p=0.244$} \\
\hline & Married & $70.0(196)$ & & $58.8(47)$ & & \\
\hline & Cohabiting & $16.4(46)$ & & $25.0(20)$ & & \\
\hline & Divorced/Separated/Widowed & $5.4(15)$ & & $7.5(6)$ & & \\
\hline \multirow[t]{4}{*}{ Location } & Dublin & $30.7(86)$ & & $28.8(23)$ & & \multirow[t]{4}{*}{$p=0.144$} \\
\hline & Cork & $7.9(22)$ & & 16.3(13) & & \\
\hline & Galway & $7.5(21)$ & & $8.8(7)$ & & \\
\hline & Rest of country & $53.9(151)$ & & $46.3(37)$ & & \\
\hline \multirow[t]{3}{*}{ No. of children } & One child & $30.4(85)$ & & 41.3(33) & & \multirow{3}{*}{$\begin{array}{l}p= \\
0.145\end{array}$} \\
\hline & Two children & $41.8(406)$ & & $38.8(31)$ & & \\
\hline & Three or more children & $27.9(78)$ & & $20.0(16)$ & & \\
\hline \multirow[t]{4}{*}{ Education } & Lower Secondary & $3.2(9)$ & & $7.5(6)$ & & \multirow{4}{*}{$\begin{array}{l}p= \\
0.360\end{array}$} \\
\hline & Upper Secondary & $12.1(34)$ & & $10.0(8)$ & & \\
\hline & Post-Secondary certificate /vocational & $26.8(75)$ & & 23.8(19) & & \\
\hline & Degree or Postgraduate & $57.9(162)$ & & $58.8(47)$ & & \\
\hline Health cover status & No cover & $11.4(32)$ & & $20.0(16)$ & & $p=0.066$ \\
\hline & Medical card only & $27.1(76)$ & & $31.3(25)$ & & \\
\hline & GP visit card only & $8.2(23)$ & & $10.0(8)$ & & \\
\hline & Private Insurance only & $35.0(98)$ & & $31.3(25)$ & & \\
\hline & Both insurance \& medical card & $18.2(51)$ & & $7.5(6)$ & & \\
\hline Healthcare professional & & $15.7(44)$ & & $8.8(7)$ & & $p=0.115$ \\
\hline $\begin{array}{l}\text { Underlying health conditions in } \\
\text { household }\end{array}$ & & $55.4(155)$ & & $55.0(44)$ & & $\begin{array}{l}p= \\
0.955\end{array}$ \\
\hline $\begin{array}{l}\text { Child under } 16 \text { with underlying } \\
\text { health condition }\end{array}$ & & $23.9(67)$ & & $22.5(18)$ & & $p=0.791$ \\
\hline Member of household isolated & & $39.6(111)$ & & $28.8(23)$ & & $p=0.075$ \\
\hline Ease of access to services & & GP & ED & GP & ED & GP: $p$ \\
\hline & Very easy & $33.6(94)$ & $18.9(53)$ & 23.8(19) & $20.0(16)$ & \\
\hline
\end{tabular}




\begin{tabular}{|c|c|c|c|c|c|c|}
\hline & Usually easy & $32.1(90)$ & $26.8(75)$ & $31.3(25)$ & 21.3(17) & \multirow[t]{4}{*}{$\begin{array}{l}\text { ED: } p= \\
0.874\end{array}$} \\
\hline & I can if I need to & $22.1(62)$ & $37.1(104)$ & $25.0(20)$ & $38.8(31)$ & \\
\hline & Somewhat difficult & 10.7(30) & $12.5(35)$ & $13.8(11)$ & $13.8(11)$ & \\
\hline & Very difficult & $1.4(4)$ & $4.6(13)$ & $6.3(5)$ & $6.3(5)$ & \\
\hline \multirow[t]{4}{*}{$\begin{array}{l}\text { Hesitancy during Contain and } \\
\text { Mitigation Stage }\end{array}$} & Much more & $28.6(80)$ & & $31.3(25)$ & & \multirow[t]{4}{*}{$\begin{array}{l}p= \\
0.460\end{array}$} \\
\hline & Somewhat more & $39.3(110)$ & & $40.0(32)$ & & \\
\hline & About the same & $24.6(69)$ & & 17.5(14) & & \\
\hline & Somewhat or much less & $7.5(21)$ & & $11.3(9)$ & & \\
\hline \multirow[t]{4}{*}{$\begin{array}{l}\text { Hesitancy during Phase one of } \\
\text { reopening }\end{array}$} & Much more & $5.4(15)$ & & $11.3(9)$ & & \multirow[t]{4}{*}{$\begin{array}{l}p= \\
0.249\end{array}$} \\
\hline & Somewhat more & $21.4(60)$ & & 22.5(18) & & \\
\hline & About the same & $41.1(115)$ & & $33.8(27)$ & & \\
\hline & Somewhat or much less & $32.1(90)$ & & $32.5(26)$ & & \\
\hline \multirow{7}{*}{$\begin{array}{l}\text { Concerns about accessing } \\
\text { healthcare during COVID }\end{array}$} & Worried about catching COVID- 19 & 66.1(185) & & $53.8(43)$ & & $p=0.044$ \\
\hline & $\begin{array}{l}\text { Worried about being judged for using a service if it } \\
\text { wasn't an emergency }\end{array}$ & $25.4(71)$ & & $27.5(22)$ & & $\begin{array}{l}p= \\
0.699\end{array}$ \\
\hline & $\begin{array}{l}\text { Thought the service would be needed more urgently by } \\
\text { other people }\end{array}$ & $18.2(51)$ & & $42.5(34)$ & & $\begin{array}{l}p> \\
0.000\end{array}$ \\
\hline & $\begin{array}{l}\text { Thought the government advice meant I couldn't go to } \\
\text { the GP or hospital }\end{array}$ & $15.0(42)$ & & $11.3(9)$ & & $\begin{array}{l}p= \\
0.396\end{array}$ \\
\hline & $\begin{array}{l}\text { Worried that the service would be extremely busy and } \\
\text { would have to wait for too long }\end{array}$ & $28.2(79)$ & & $20.0(16)$ & & $\begin{array}{l}p= \\
0.142\end{array}$ \\
\hline & Don't have a car and didn't want to use public transport & $7.1(20)$ & & $6.3(5)$ & & $p=0.782$ \\
\hline & Something else & $11.1(31)$ & & $15.0(12)$ & & $\begin{array}{l}p= \\
0.339\end{array}$ \\
\hline \multirow[t]{4}{*}{$\begin{array}{l}\text { Perception of Risk of child } \\
\text { contracting COVID }\end{array}$} & High risk & $12.5(35)$ & & 11.3(9) & & \multirow[t]{4}{*}{$\begin{array}{l}p= \\
0.842\end{array}$} \\
\hline & Moderately concerned & $31.4(88)$ & & $31.3(25)$ & & \\
\hline & Slightly concerned & $40.4(113)$ & & $45.0(36)$ & & \\
\hline & Not at all & $15.7(141)$ & & $12.5(10)$ & & \\
\hline \multirow{4}{*}{$\begin{array}{l}\text { Perception of effect of COVID on } \\
\text { child }\end{array}$} & Very unwell & $12.9(36)$ & & 11.3(9) & & \multirow[t]{4}{*}{$p=0.960$} \\
\hline & Quite unwell & 29.3(82) & & $31.3(25)$ & & \\
\hline & Somewhat unwell & $45.4(127)$ & & $46.3(37)$ & & \\
\hline & Unaffected & $12.5(35)$ & & $11.3(9)$ & & \\
\hline \multirow[t]{5}{*}{ DASS Stress } & Normal & $50.7(142)$ & & $56.3(45)$ & & \multirow{5}{*}{$\begin{array}{l}p= \\
0.291\end{array}$} \\
\hline & Mild & $19.6(55)$ & & $16.3(55)$ & & \\
\hline & Moderate & $17.1(48)$ & & $13.8(11)$ & & \\
\hline & Severe & $9.3(26)$ & & $13.8(11)$ & & \\
\hline & Extremely severe & $3.2(9)$ & & $0.0(0)$ & & \\
\hline
\end{tabular}

\section{Regression Analyses}

For the purpose of regression analysis, some categorical variables were collapsed to ensure adequate sample size and to aid interpretation, such as age of parent (under 40/ over 40), education (below degree/degree post grad), concern of effect of COVID-19 on child (somewhat unwell or unaffected/ very or quite unwell), perception of risk of child contracting COVID -19 (slightly concerned or not at all/ highly or moderately concerned) and easy access to the GP or ED (very or usually easy/ can if I have to, somewhat or very difficult). For the DASS stress categories, mild and moderate stress were collapsed into one category and severe and extremely severe stress were also collapsed into one category. Details of these groups can be viewed in Additional File 3. 


\section{Hesitancy to attend health services}

The results from a multinomial regression estimating the association between reported hesitancy to attend health services and demographic, health, and COVID-related characteristics are represented in Table 4. Females were found to be twice as likely to report being much more hesitant during the Delay and Mitigation stage (RRR= 194, Cl: 1.33 - 2.82). Higher than normal stress levels were also significantly associated with hesitancy. Those who reported mild/ moderate stress were over twice as likely to report being much more hesitant (RRR=2.31, Cl: 1.54 - 3.47), while those with severe/extremely severe stress were over three times more likely (RRR:3.37, Cl:1.81 - 6.27). Finally, those who felt that the government advice was to stay away from health services were 1.7 times more likely to be much more hesitant (RRR:1.71, Cl; $1.10-2.67)$.

In Phase One of reopening, while fewer respondents were much more or somewhat more hesitant, those with higher than normal stress levels were more likely to report hesitancy than in the Delay and Mitigation phase than those with normal stress levels. Respondents who reported having severe or extremely severe stress levels were over five times more likely to be much more hesitant (RRR: 5.22, Cl: 2.22-12.29), while those with mild or moderate stress levels were over three times more likely (RRR: 3.10, Cl: 1.56 - 6.20). Similarly, those who felt government advice was to stay away had a slightly higher likelihood of reporting being much more hesitant in Phase One (RRR: 2.19, Cl: 1.12 - 4.29). Parents who accessed healthcare for their child during the Delay and Mitigation phase were no more or less hesitant about accessing during Phase One. 
Table 4

Multinomial Regression Results; Reported Hesitancy in both Lockdown and Phase One periods $N=1044$

\begin{tabular}{|c|c|c|c|c|c|c|}
\hline \multirow[t]{2}{*}{ Ref: About the same } & \multicolumn{3}{|c|}{ Delay \& Mitigation Period ("Lockdown") } & \multicolumn{3}{|c|}{ Phase One of reopening } \\
\hline & $\begin{array}{l}\text { Much } \\
\text { more }\end{array}$ & $\begin{array}{l}\text { Somewhat } \\
\text { more }\end{array}$ & $\begin{array}{l}\text { Somewhat or } \\
\text { much less }\end{array}$ & $\begin{array}{l}\text { Much } \\
\text { more }\end{array}$ & $\begin{array}{l}\text { Somewhat } \\
\text { more }\end{array}$ & $\begin{array}{l}\text { Somewhat or much } \\
\text { less }\end{array}$ \\
\hline \multirow[t]{2}{*}{ Gender: Female } & $1.94 * \star$ & $1.37 *$ & 0.71 & 1.33 & 1.16 & $1.67 * \star$ \\
\hline & $\begin{array}{l}(1.33- \\
2.82)\end{array}$ & $(1.00-1.88)$ & $(0.43-1.20)$ & $\begin{array}{l}(0.71- \\
2.48)\end{array}$ & $(0.80-1.67)$ & $(1.23-2.28)$ \\
\hline \multirow[t]{2}{*}{ Participant Age: Under 40} & 0.90 & 1.01 & 1.53 & 0.79 & 1.25 & 0.82 \\
\hline & $\begin{array}{l}(0.63- \\
1.29)\end{array}$ & $(0.74-1.38)$ & $(0.89-2.61)$ & $\begin{array}{l}(0.41- \\
1.50)\end{array}$ & $(0.86-1.82)$ & $(0.61-1.12)$ \\
\hline \multirow[t]{2}{*}{ Marital Status: Married or cohabiting } & 0.95 & 1.38 & 1.57 & 0.60 & 1.06 & 0.77 \\
\hline & $\begin{array}{l}(0.59- \\
1.52)\end{array}$ & $(0.90-2.13)$ & $(0.75-3.30)$ & $\begin{array}{l}(0.27- \\
1.32)\end{array}$ & $(0.64-1.77)$ & $(0.51-1.16)$ \\
\hline \multirow[t]{2}{*}{ Education: Degree or Post-graduate } & 1.14 & 1.17 & 0.95 & 0.90 & 0.98 & 0.87 \\
\hline & $\begin{array}{l}(0.79- \\
1.65)\end{array}$ & $(0.86-1.61)$ & $(0.56-1.63)$ & $\begin{array}{l}(0.48- \\
1.68)\end{array}$ & $(0.67-1.41)$ & $(0.64-1.19)$ \\
\hline \multicolumn{7}{|l|}{ Health Cover (ref: no cover) } \\
\hline \multirow[t]{2}{*}{ Medical card only } & 0.86 & 0.99 & $2.52^{\star}$ & 1.00 & 0.90 & $0.64^{*}$ \\
\hline & $\begin{array}{l}(0.50- \\
1.46)\end{array}$ & $(0.63-1.55)$ & $(1.14-5.57)$ & $\begin{array}{l}(0.35- \\
2.88)\end{array}$ & $(0.52-1.54)$ & $(0.41-0.99)$ \\
\hline \multirow[t]{2}{*}{ GP visit card only } & 1.70 & 1.65 & 2.52 & 0.73 & 1.14 & 0.70 \\
\hline & $\begin{array}{l}(0.81- \\
3.57)\end{array}$ & $(0.87-3.14)$ & $(0.87-7.28)$ & $\begin{array}{l}(0.14- \\
3.90)\end{array}$ & $(0.56-2.33)$ & $(0.38-1.31)$ \\
\hline \multirow[t]{2}{*}{ Health insurance } & 1.29 & 1.20 & 1.11 & 1.95 & 1.06 & 1.09 \\
\hline & $\begin{array}{l}(0.79- \\
2.13)\end{array}$ & $(0.79-1.84)$ & $(0.48-2.60)$ & $\begin{array}{l}(0.73- \\
5.15)\end{array}$ & $(0.63-1.79)$ & $(0.73-1.65)$ \\
\hline \multirow[t]{2}{*}{ Health insurance and Medical card } & 1.22 & 1.42 & 2.62 & 3.14 & 1.27 & 0.83 \\
\hline & $\begin{array}{l}(0.60- \\
2.50)\end{array}$ & $(0.77-2.63)$ & $(0.93-7.32)$ & $\begin{array}{l}(0.98- \\
10.10)\end{array}$ & $(0.64-2.55)$ & $(0.45-1.54)$ \\
\hline \multirow[t]{2}{*}{ Healthcare worker } & 0.87 & 0.90 & 1.23 & 2.15 & 1.15 & 0.83 \\
\hline & $\begin{array}{l}(0.48- \\
1.58)\end{array}$ & $(0.54-1.49)$ & $(0.55-2.75)$ & $\begin{array}{l}(0.89- \\
5.20)\end{array}$ & $(0.65-2.03)$ & $(0.50-1.38)$ \\
\hline \multicolumn{7}{|c|}{ No. of Children (ref: three children or more) } \\
\hline \multirow[t]{2}{*}{ One child } & 0.72 & $0.49 * *$ & $0.33^{* *}$ & 1.04 & 0.89 & $0.57 * \star$ \\
\hline & $\begin{array}{l}(0.44- \\
1.17)\end{array}$ & $(0.32-0.75)$ & $(0.17-0.65)$ & $\begin{array}{l}(0.45- \\
2.40)\end{array}$ & $(0.55-1.43)$ & $(0.38-0.85)$ \\
\hline \multirow[t]{2}{*}{ Two children } & 0.85 & $0.64^{*}$ & 0.56 & 1.09 & 0.91 & 0.78 \\
\hline & $\begin{array}{l}(0.52- \\
1.39)\end{array}$ & $(0.42-0.98)$ & $(0.30-1.06)$ & $\begin{array}{l}(0.47- \\
2.53)\end{array}$ & $(0.57-1.47)$ & $(0.53-1.14)$ \\
\hline \multirow[t]{2}{*}{ Health condition household } & 1.08 & 0.99 & 1.09 & 0.63 & 1.07 & 1.22 \\
\hline & $\begin{array}{l}(0.72- \\
1.63)\end{array}$ & $(0.70-1.41)$ & $(0.61-1.95)$ & $\begin{array}{l}(0.31- \\
1.28)\end{array}$ & $(0.71-1.61)$ & $(0.87-1.72)$ \\
\hline \multirow[t]{2}{*}{ Health condition under 16 child(ren) } & 1.45 & 1.34 & 0.75 & 0.36 & 0.76 & 0.77 \\
\hline & $\begin{array}{l}(0.83- \\
2.51)\end{array}$ & $(0.81-2.22)$ & $(0.32-1.79)$ & $\begin{array}{l}(0.11- \\
1.11)\end{array}$ & $(0.44-1.32)$ & $(0.48-1.23)$ \\
\hline \multicolumn{7}{|l|}{ DASS Stress (ref: normal) } \\
\hline \multirow[t]{2}{*}{ Mild/moderate } & $2.31 * \star \star$ & $1.46 *$ & 1.03 & $3.10 * \star$ & $1.91^{\star *}$ & 1.34 \\
\hline & $\begin{array}{l}(1.54- \\
3.47)\end{array}$ & $(1.01-2.10)$ & $(0.55-1.92)$ & $\begin{array}{l}(1.56- \\
6.20)\end{array}$ & $(1.27-2.87)$ & $(0.94-1.91)$ \\
\hline \multirow[t]{2}{*}{ Severe/extremely severe } & $3.37^{\star \star \star}$ & 1.48 & 1.18 &  & 1.80 & 1.12 \\
\hline & $\begin{array}{l}(1.81- \\
6.27)\end{array}$ & $(0.79-2.77)$ & $(0.43-3.22)$ & $\begin{array}{l}(2.22- \\
12.29)\end{array}$ & $(0.98-3.30)$ & $(0.63-1.99)$ \\
\hline
\end{tabular}




\begin{tabular}{|c|c|c|c|c|c|c|}
\hline \multirow[t]{2}{*}{ Very or quite concerned effect COVID on child } & 1.45 & 0.91 & 1.09 & 1.44 & 1.04 & $0.68^{*}$ \\
\hline & $\begin{array}{l}(0.98- \\
2.13)\end{array}$ & $(0.65-1.29)$ & $(0.63-1.90)$ & $\begin{array}{l}(0.74- \\
2.79)\end{array}$ & $(0.70-1.53)$ & $(0.49-0.96)$ \\
\hline \multirow{2}{*}{$\begin{array}{l}\text { High risk/moderately concerned risk contracting } \\
\text { COVID child }\end{array}$} & $1.85^{\star \star}$ & 1.22 & $1.87 *$ & $2.78 * \star$ & $1.87^{\star \star}$ & 1.24 \\
\hline & $\begin{array}{l}(1.27- \\
2.70)\end{array}$ & $(0.87-1.71)$ & $(1.09-3.22)$ & $\begin{array}{l}(1.43- \\
5.40)\end{array}$ & $(1.28-2.73)$ & $(0.90-1.72)$ \\
\hline \multirow[t]{2}{*}{ Gov advice stay away } & $1.71^{\star}$ & 1.17 & 0.75 & $2.19 *$ & 1.19 & 1.01 \\
\hline & $\begin{array}{l}(1.10- \\
2.67)\end{array}$ & $(0.77-1.78)$ & $(0.35-1.59)$ & $\begin{array}{l}(1.12- \\
4.29)\end{array}$ & $(0.76-1.87)$ & $(0.68-1.51)$ \\
\hline \multirow[t]{2}{*}{ Easy access to GP } & 1.15 & 1.09 & 0.80 & 1.51 & 1.30 & 1.09 \\
\hline & $\begin{array}{l}(0.77- \\
1.72)\end{array}$ & $(0.77-1.54)$ & $(0.46-1.41)$ & $\begin{array}{l}(0.74- \\
3.08)\end{array}$ & $(0.87-1.95)$ & $(0.79-1.52)$ \\
\hline \multirow[t]{2}{*}{ Easy access to ED } & 1.09 & 0.97 & 0.91 & 1.18 & 1.11 & 0.94 \\
\hline & $\begin{array}{l}(0.74- \\
1.60)\end{array}$ & $(0.70-1.36)$ & $(0.51-1.60)$ & $\begin{array}{l}(0.61- \\
2.29)\end{array}$ & $(0.76-1.64)$ & $(0.68-1.29)$ \\
\hline \multirow[t]{2}{*}{ Household member self-isolated } & 1.17 & 1.24 & 0.70 & 1.13 & 1.10 & 1.00 \\
\hline & $\begin{array}{l}(0.77- \\
1.79)\end{array}$ & $(0.85-1.81)$ & $(0.36-1.36)$ & $\begin{array}{l}(0.55- \\
2.34)\end{array}$ & $(0.72-1.69)$ & $(0.70-1.45)$ \\
\hline \multirow[t]{2}{*}{ Accessed healthcare } & & & & 0.96 & 1.24 & 1.24 \\
\hline & & & & $\begin{array}{l}(0.46- \\
2.00)\end{array}$ & $(0.82-1.87)$ & $(0.87-1.76)$ \\
\hline \multicolumn{7}{|l|}{$*=p<.05$} \\
\hline \multicolumn{7}{|l|}{$\star *=p<.01$} \\
\hline$\star \star \star=p<.001$ & & & & & & \\
\hline
\end{tabular}

\section{Official public health advice to avoid health services}

Logistic regressions were carried out to examine the factors that contributed to the understanding that government advice was to stay away from health services. The results are reported in Table 5.

When using the full sample $(\mathrm{N}=1,044)$, those who reported mild or moderate stress levels were 1.7 times more likely to hold this perception (OR: 1.66 , Cl: 1.14 - 2.41), while those with severe or extremely severe stress were nearly twice as likely (OR: 1.87, Cl: $1.10-3.20$ ). Looking specifically at the group who needed to access healthcare $(n=360)$, those that had mild or moderate stress levels were 2.4 times more likely to have reported that they thought government advice was to stay away (OR: $2.36, \mathrm{Cl}: 1.12-4.99)$. Those that reported severe or extremely severe stress were also more likely to hold this perception, however this was not significant (OR: 2.29 , Cl: $0.87-6.07$ ). Those that perceived their child to be at high or moderate risk of contracting COVID-19 were 3.4 times more likely to believe the official public advice was to avoid healthcare services (OR:3.40, Cl:1.59-7.28). On the other hand, those that were very or quite concerned about the effect COVID-19 would have on their child were less likely to hold this view (OR: 0.43, Cl:0.20-0.94). 
Table 1

Odds Ratio from Logistic Regression

\begin{tabular}{|c|c|c|}
\hline & $\begin{array}{l}\text { Believed government advice was to stay } \\
\text { away }(n=1,044)\end{array}$ & $\begin{array}{l}\text { Believed government advice was to stay away, healthcare } \\
\text { needed only }(n=360)\end{array}$ \\
\hline & ODDS Ratio (95\% conf. Interval) & ODDS Ratio (95\% conf. Interval) \\
\hline \multirow[t]{2}{*}{ Gender: Female } & 0.96 & 1.43 \\
\hline & $(0.69-1.36)$ & $(0.69-2.96)$ \\
\hline \multirow[t]{2}{*}{ Participant Age: Under 40} & 1.18 & 1.83 \\
\hline & $(0.83-1.66)$ & $(0.84-3.97)$ \\
\hline \multirow[t]{2}{*}{ Marital Status: Married or cohabiting } & 1.45 & 0.94 \\
\hline & $(0.89-2.37)$ & $(0.36-2.48)$ \\
\hline \multirow[t]{2}{*}{ Education: Degree or Post-graduate } & 1.39 & 1.50 \\
\hline & $(0.98-1.97)$ & $(0.73-3.09)$ \\
\hline \multicolumn{3}{|l|}{ Health cover } \\
\hline \multirow[t]{2}{*}{ Medical card only } & 1.24 & 1.95 \\
\hline & $(0.74-2.06)$ & $(0.55-6.83)$ \\
\hline \multirow[t]{2}{*}{ GP visit card only } & 1.11 & 2.00 \\
\hline & $(0.55-2.25)$ & $(0.40-9.91)$ \\
\hline \multirow[t]{2}{*}{ Health insurance } & 1.05 & 1.52 \\
\hline & $(0.64-1.71)$ & $(0.42-5.46)$ \\
\hline \multirow[t]{2}{*}{ Health insurance and Medical card } & 1.32 & 3.13 \\
\hline & $(0.69-2.52)$ & $(0.83-11.82)$ \\
\hline \multirow[t]{2}{*}{ Healthcare worker } & 0.64 & 0.83 \\
\hline & $(0.36-1.16)$ & $(0.32-2.12)$ \\
\hline \multicolumn{3}{|l|}{ No. of Children: (ref: three children or more) } \\
\hline \multirow[t]{2}{*}{ One child } & 1.37 & 1.14 \\
\hline & $(0.87-2.16)$ & $(0.48-2.68)$ \\
\hline \multirow[t]{2}{*}{ Two children } & 1.08 & 0.97 \\
\hline & $(0.68-1.71)$ & $(0.43-2.19)$ \\
\hline \multirow[t]{2}{*}{ Health condition household } & 1.10 & $2.69 *$ \\
\hline & $(0.75-1.60)$ & $(1.21-5.98)$ \\
\hline \multirow[t]{2}{*}{ Health condition under 16 child(ren) } & 0.86 & 0.85 \\
\hline & $(0.51-1.46)$ & $(0.37-1.95)$ \\
\hline \multicolumn{3}{|l|}{ DASS Stress: (ref: normal) } \\
\hline \multirow[t]{2}{*}{ Mild/moderate } & $1.66^{\star \star}$ & $2.36^{*}$ \\
\hline & $(1.14-2.41)$ & $(1.12-4.99)$ \\
\hline \multirow[t]{2}{*}{ Severe/extremely severe } & $1.87^{\star}$ & 2.29 \\
\hline & $(1.10-3.20)$ & $(0.87-6.07)$ \\
\hline \multirow[t]{2}{*}{ Very or quite concerned effect COVID on child } & 0.89 & $0.43^{*}$ \\
\hline & $(0.62-1.29)$ & $(0.20-0.94)$ \\
\hline \multirow{2}{*}{$\begin{array}{l}\text { High risk/moderately concerned risk } \\
\text { contracting COVID child }\end{array}$} & 1.28 & $3.40 \star *$ \\
\hline & $(0.89-1.82)$ & $(1.59-7.28)$ \\
\hline \multirow[t]{2}{*}{ Household member self-isolated } & 0.99 & 0.72 \\
\hline & $(0.67-1.48)$ & $(0.34-1.52)$ \\
\hline Easy access to GP & $0.68^{*}$ & 0.84 \\
\hline
\end{tabular}

Page 14/17 
$*=p<.05$

$\star *=p<.01$

$\star \star *=p<.001$

\section{Discussion}

This study sought to capture parents' experiences of seeking healthcare for their children during the implementation of public health measures and restrictions to delay the spread of COVID-19. Delayed presentation or avoidance of the emergency department during the COVID-19 pandemic has been a cause for concern $(7,8)$, however, there is little understanding of the characteristics of those that have avoided or would be hesitant to attend health services. Approximately one third of the present sample required healthcare for their children during the public health restrictions to delay the spread of COVID-19 but one in five of these parents avoided accessing such healthcare when needed. Of those that required healthcare, parents who avoided were more likely report that the services were needed more by others. The survey also assessed the degree to which parents were hesitant to attend health services for their children during both the most severe and limiting restrictions (i.e., Delay and Mitigation period) and during the first step in the plan to ease the restrictions (i.e., Phase One). Factors that influenced the extent to which parents were hesitant included higher than normal levels of stress and their perception of the risk of their child contracting COVID-19. Moreover, the misinterpretation of the government's public health advice also played a role in whether parents were more likely to be hesitant.

While there was significant reliance on official government advice, a key finding in the study was that a proportion of the sample may have misunderstood this government advice which may have resulted in unnecessary avoidance of or hesitancy to access health services. The unprecedented nature of this global pandemic has resulted in the release of a substantial amount of information into the public domain, which has necessitated the need for clear and tailored public health messaging from health authorities and government bodies (12). Nevertheless, even clear and coherent messaging around the importance of hand-washing and maintaining physical distancing, coupled with the perception of the risk of COVID-19, requires the ability to obtain, understand and use this information to make informed decisions (13). Such health literacy is vital in the maintenance of public health, however, social determinants of health literacy can significantly disadvantage particular sections of the population (14) which can exacerbate existing health inequalities (15). Moreover, economic uncertainty and social isolation may increase anxiety and stress (15) and in the present study, stress was found to be a significant driver of hesitancy and misunderstanding of public health advice. Public health officials need to be cognizant of the unintended consequences of the messages designed to inform the public as the pandemic progresses and during future public health emergencies.

Understanding the public perceptions of the risk of contracting COVID-19 is important for the effectiveness and success of government responses to control the disease (16) and in the current study, a greater perception of the risk that their child would contract COVID-19 was linked to hesitancy to attend health services. Public compliance is required for wide-ranging public health strategies such as physical distancing, hand hygiene, closure of schools and nonessential workplaces to be effective $(1,16)$ and the use of formal information sources are critical for encouraging protective behaviours such as social distancing (17). However, it is important for public health messaging and health services to adequately communicate to the public that essential services such as health remain open and are safe to attend when needed to avoid deterioration of a child's illness (4). The decision to seek health care is complex (18) and current circumstances require parents to weigh up information regarding their child's need for healthcare for a non-COVID-19 related illness, the risk of their child catching COVID-19 and the government public heath advice. Moreover, experience of COVID-19 may also impact their perception of this risk involved (16), however, only a small number of the current sample had personal experience of the disease. It is worth noting that a commonly reported reason for the drastic public measures was the need to maintain capacity in health services for the most vulnerable members of the population (13). This may have been related in the present survey where many parents stated that they felt that services were needed more by others or that they did not want to burden the health system at such a time.

\section{Limitations}

The sample recruited to the present study was largely representative of the general population on factors such as education and health insurance status, and there was an adequate spread across age groups and geographical region. Females were over-represented in the sample, however, this is common in studies of parental behaviour. Data collection occurred during the first step of lifting restrictions and therefore, reporting on hesitancy during the Delay and Mitigation phase was somewhat retrospective. As specific information on the clinical reason for requiring healthcare was not collected, we have no information on the severity of the illness for those who accessed or avoided healthcare. However, understanding when and why patients seek healthcare is highly complex and requires in-depth knowledge of how individuals perceive their health and health services (19) and thus, this information may not have provided more context to the present results.

\section{Conclusion}

The present study sought to understand parents' experiences of healthcare during the COVID-19 pandemic and aimed to provide context as to why some parents may have avoided or were hesitant to access healthcare during this time. Government public health messaging, stress and the perception of risk each contributed to parental avoidance of or hesitancy to utilize healthcare services during the public health measures imposed to combat COVID-19. The longerterm impact of such avoidance remains unknown with regards to clinical outcomes and mortality for non-COVID-19 conditions. 


\section{Declarations}

\section{Ethics approval and consent to participate}

The study was granted full ethical approval by the COVID-19 National Research Ethics Committee (NREC) in Ireland (ref: 20-NREC-COV-034). All participants provided informed consent online prior to participating in the survey.

\section{Consent for publication}

Not applicable

\section{Availability of data and materials}

The datasets used and/or analysed during the current study are available from the corresponding author on reasonable request.

\section{Competing interests}

The authors declare that they have no competing interests.

\section{Funding}

The study was funded by the Health Research Board (HRB) (Grant Number: COV19-2020-076). The funder had no role in the study.

\section{Authors' contributions}

EN, TMcD, CC designed and conducted the study. EN prepared the manuscript. TMcD, CC, MB, FC, CH, EMcA provided feedback and approved the final manuscript.

\section{Acknowledgements}

Not Applicable

\section{References}

1. Prem K, Liu Y, Russell TW, Kucharski AJ, Eggo RM, Davies N, et al. The effect of control strategies to reduce social mixing on outcomes of the COVID-19 epidemic in Wuhan, China: a modelling study. The Lancet Public Health. 2020;5(5):e261-e70.

2. Blendon RJ, Benson JM, DesRoches CM, Raleigh E, Taylor-Clark K. The Public's Response to Severe Acute Respiratory Syndrome in Toronto and the United States. Clin Infect Dis. 2004;38(7):925-31.

3. Sadique MZ, Edmunds WJ, Smith RD, Meerding WJ, de Zwart O, Brug J, et al. Precautionary behavior in response to perceived threat of pandemic influenza. Emerg Infect Dis. 2007;13(9):1307-13.

4. Lazzerini M, Barbi E, Apicella A, Marchetti F, Cardinale F, Trobia G. Delayed access or provision of care in Italy resulting from fear of COVID-19. The Lancet Child adolescent health. 2020;4(5):e10-e1.

5. Dann L, Fitzsimons J, Gorman KM, Hourihane J, Okafor I. Disappearing act: COVID-19 and paediatric emergency department attendances. Archives of disease in childhood. 2020:archdischild-2020-319654.

6. Isba R, Edge R, Jenner R, Broughton E, Francis N, Butler J. Where have all the children gone? Decreases in paediatric emergency department attendances at the start of the COVID-19 pandemic of 2020. Arch Dis Child. 2020;105(7):704-

7. Lynn RM, Avis JL, Lenton S, Amin-Chowdhury Z, Ladhani SN. Delayed access to care and late presentations in children during the COVID-19 pandemic: a snapshot survey of 4075 paediatricians in the UK and Ireland. Archives of disease in childhood. 2020:archdischild-2020-319848.

8. Roland D, Harwood R, Bishop N, Hargreaves D, Patel S, Sinha I. Children's emergency presentations during the COVID-19 pandemic. The Lancet Child \& Adolescent Health. 2020.

9. Lovibond PF, Lovibond SH. The structure of negative emotional states: Comparison of the Depression Anxiety Stress Scales (DASS) with the Beck Depression and Anxiety Inventories. Behav Res Ther. 1995;33(3):335-43.

10. Department of Health. Health in Ireland Key Trends 2019. Dublin, Ireland Department of Health; 2019.

11. Health Insurance Authority. Market Statistics 2020 [cited 2020 March ]. Available from: https://www.hia.ie/publication/market-statistics.

12. Zarocostas J. How to fight an infodemic. The Lancet. 2020;395(10225):676.

13. Paakkari L, Okan O. COVID-19: health literacy is an underestimated problem. The Lancet Public Health. 2020;5(5):e249-e50. 
14. Rikard RV, Thompson MS, McKinney J, Beauchamp A. Examining health literacy disparities in the United States: a third look at the National Assessment of Adult Literacy (NAAL). BMC Public Health. 2016;16(1):975.

15. Bavel JJV, Baicker K, Boggio PS, Capraro V, Cichocka A, Cikara M, et al. Using social and behavioural science to support COVID-19 pandemic response. Nature Human Behaviour. 2020;4(5):460-71.

16. Dryhurst S, Schneider CR, Kerr J, Freeman ALJ, Recchia G, van der Bles AM, et al. Risk perceptions of COVID-19 around the world. Journal of Risk Research. 2020:1-13.

17. Qazi A, Qazi J, Naseer K, Zeeshan M, Hardaker G, Maitama JZ, et al. Analyzing situational awareness through public opinion to predict adoption of social distancing amid pandemic COVID-19. J Med Virol. 2020;92(7):849-55.

18. Nicholson E, McDonnell T, De Brún A, Barrett M, Bury G, Collins C, et al. Factors that influence family and parental preferences and decision making for unscheduled paediatric healthcare - systematic review. BMC Health Serv Res. 2020;20(1):663.

19. Pope C, McKenna G, Turnbull J, Prichard J, Rogers A. Navigating and making sense of urgent and emergency care processes and provision. Health expectations: an international journal of public participation in health care health policy. 2019;22(3):435-43.

\section{Figures}

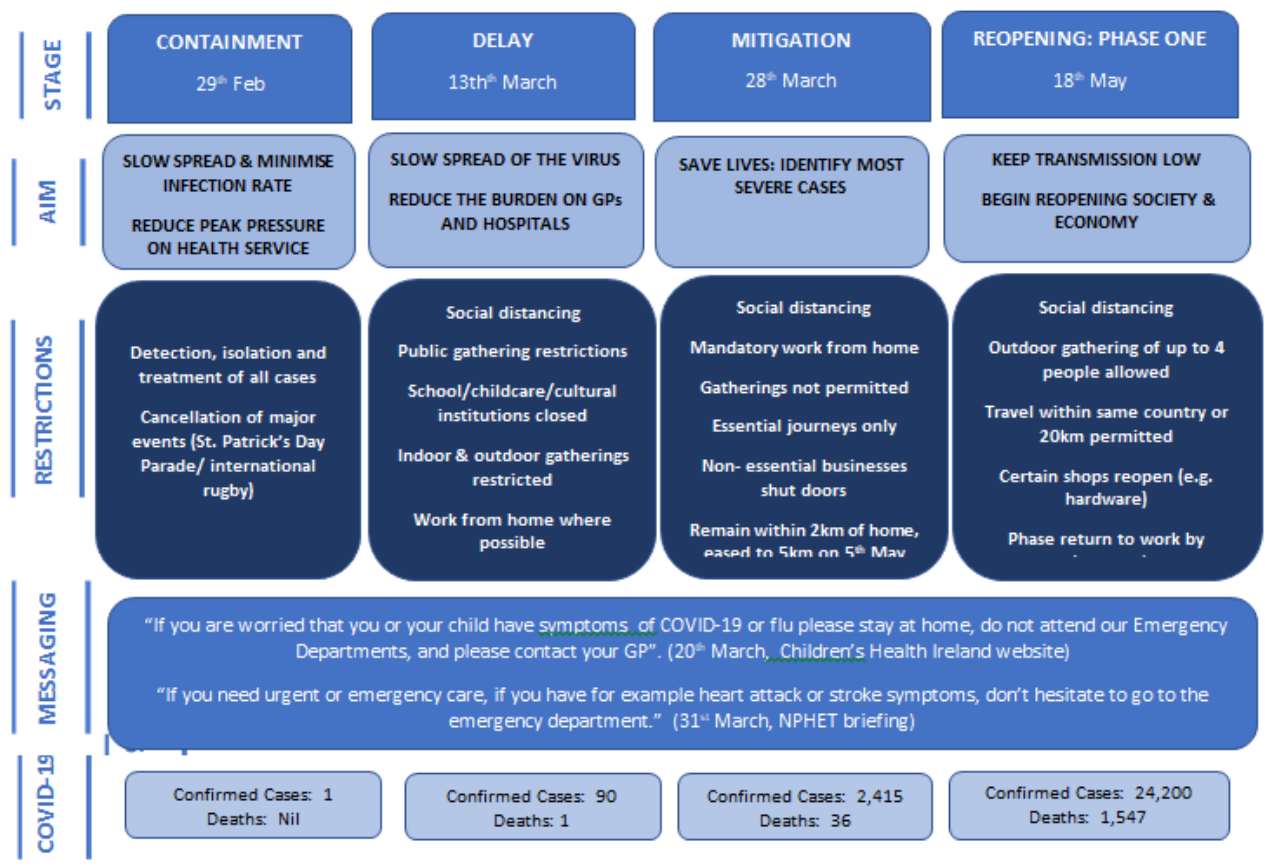

\section{Figure 1}

Public health stages of COVID-19 response

\section{Supplementary Files}

This is a list of supplementary files associated with this preprint. Click to download.

- STROBEchecklistcrosssectional.doc

- AdditionalFile3.docx

- AdditionalFile2.docx

- AdditionalFile1.docx 